\title{
Transport urbain au Benin et pollution atmosphérique: évaluation quantitative de certains polluants chimiques de Cotonou
}

\author{
Daouda MAMA ${ }^{1,2^{*}}$, Biaou DIMON ${ }^{2}$, Martin AINA ${ }^{1,2}$, Julien ADOUNKPE $^{4}$, \\ Mathias AHOMADEGBE ${ }^{2}$, Alassane YOUSSAO ${ }^{2}$, Jacques KOUAZOUNDE ${ }^{2}$, \\ Séïdou PEDRO KOUANDA ${ }^{3}$ et Mansourou MOUDACHIROU ${ }^{2}$ \\ ${ }^{1}$ Laboratoire d'Hydrologie Appliquée (LHA) Faculté des Sciences Techniques (FAST) Université d'Abomey- \\ Calavi (UAC) 01 BP 526 Cotonou, Bénin. \\ ${ }^{2}$ Laboratoire d'Expertise et de Recherche en Chimie de l'Eau et l'Environnement (LERCEE) Département de \\ Chimie, / Faculté des Sciences et Techniques (FAST) Université d'Abomey - Calavi (UAC) 01 BP 526 \\ Cotonou, Bénin. \\ ${ }^{3}$ Société Plastique et élastomère du Bénin (PEB) Cotonou, Bénin. \\ ${ }^{4}$ Laboratoire d'Ecologie Appliquée, Faculté des Sciences Agronomique (FSA) Université d'Abomey - Calavi \\ (UAC) 01 BP 526 Cotonou, Bénin. \\ *Auteur correspondant, E-mail : mkdaouda@yahoo.fr
}

\section{RESUME}

L'atmosphère dans la ville de Cotonou au Bénin en Afrique de l'Ouest, se caractérise par la présence de poussières soulevées au cours de la circulation sur toutes les voies et presque partout dans cette ville, par d'épaisses fumées opaques, en particulier au niveau des grandes artères et des carrefours. Ce phénomène est lié à une utilisation des moto à deux roues notamment les taxis-moto appelés «Zémidjan » dont le nombre en 2005 est estimé à 96.095 selon les statistiques de la mairie de Cotonou. Le présent article vise l'évaluation quantitative de certains polluants chimiques supposés nocifs et ayant fait l'objet de réglementation au niveau du Bénin. Il met en relief le rôle joué par le transport dans l'émission de ces polluants gazeux. A cet effet, les taux dans l'air du monoxyde de carbone $(\mathrm{CO})$, du dioxyde d'azote $\left(\mathrm{NO}_{2}\right)$, du dioxyde de soufre $\left(\mathrm{SO}_{2}\right)$ et du plomb ont été déterminés. Le monoxyde de carbone et le dioxyde d'azote donnent des taux dépassant souvent le seuil retenu par la réglementation Béninoise. Le carrefour du grand marché Tokpa de Cotonou présente les plus forts taux mesurés avec un maximum de $214,7 \mathrm{mg} / \mathrm{m}^{3}$ de CO. La quantité du plomb fixée varie avec le temps d'exposition. Le travail a confirmé le rôle principal de pollution de l'air joué par le transport urbain à Cotonou.

(C) 2013 International Formulae Group. All rights reserved.

Mots clés: Transport urbain, Pollution atmosphérique, Evaluation quantitative, polluants chimiques, Cotonou.

$\begin{aligned} & \text { INTRODUCTION } \\ & \text { La pollution atmosphérique est }\end{aligned}$
$\begin{aligned} & \text { manières. Certaines des conséquences } \\ & \text { engendrées sont mesurables par exemple, } \\ & \text { celles qui ont lieu vis-à-vis des sols (c'est le }\end{aligned}$
aujourd'hui l'une des grandes causes des
problèmes de santé publique. Les polluants
atmosphériques peuvent exercer une influence la teneur en métaux lourds). D'autres
néfaste sur l'atmosphère de différentes


hommes particulièrement celle des enfants (cas du plomb) dont le système immunitaire n'est pas encore complètement développé (PNUE, 1987; David et Zali, 1992; WHO, 1999). Cette pollution n'est pas sans effet sur les végétaux et les matériaux ; rien n'est donc épargné par le désastre. Les effets sur les changements climatiques illustrent ce fait dans la mesure où ces effets se font sentir durablement. (Avognon-Houéto, 1997 ; Moudachirou, 2005).

Depuis une dizaine d'années, il est aisé de constater que l'atmosphère dans la ville de Cotonou est de plus en plus dégradée. En effet, outre les poussières soulevées par la circulation sur toutes les voies, l'on observe presque partout dans cette ville d'épaisses fumées opaques, en particulier au niveau des grandes artères et des carrefours équipés de feux tricolores.

Dans le cadre de cette contribution à l'étude de la pollution de l'air dans la ville de Cotonou, nous avons focalisé notre attention sur l'évaluation quantitative de certains polluants chimiques supposés nocifs et ayant fait l'objet de réglementation au niveau du Bénin. La réalisation des mesures quantitatives des polluants que sont le $\mathrm{CO}$ le $\mathrm{NO}_{2}$ le $\mathrm{SO}_{2}$ et le plomb dans l'air ambiant et sous abri. Les taux des polluants ont été évalués au plan spatial et en dynamique.

L'appréciation de l'évolution de la pollution atmosphérique dans la ville et son lien avec le transport ont été abordés.

\section{MATERIEL ET METHODES}

\section{Le transport terrestre à Cotonou}

Pour une ville de $79 \mathrm{~km}^{2}$, où est concentrée la grande partie des activités administratives, économiques, et politiques du pays; le transport terrestre joue un rôle primordial. Son accroissement varie parallèlement avec celui de la démographie estimée à $5 \%$ par an. Le parc automobile de Cotonou représente $36 \%$ environ de celui du pays.

Cette concentration de motocycles et de véhicules n'est pas sans effet sur la fluidité du trafic, rendant ainsi très difficile la circulation aux heures de pointe. Pour s'en convaincre, il n'y a qu'à observer le flux au niveau des feux tricolores, certains carrefours et principaux axes de la ville comme le montre la Photo 1. La vente du carburant de contrebande en provenance du Nigéria occupe les artères de la ville de Cotonou avec tout son corolaire de risques (incendie et intoxication par inhalation) comme le montre la Photo 2.

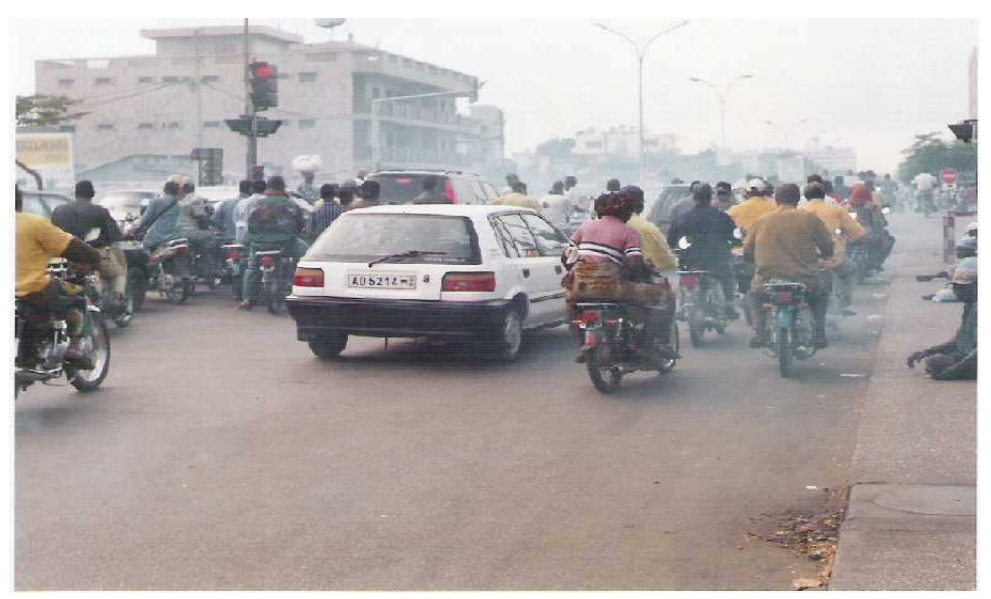

Photo 1: Le trafic à une heure de pointe à Cotonou. 


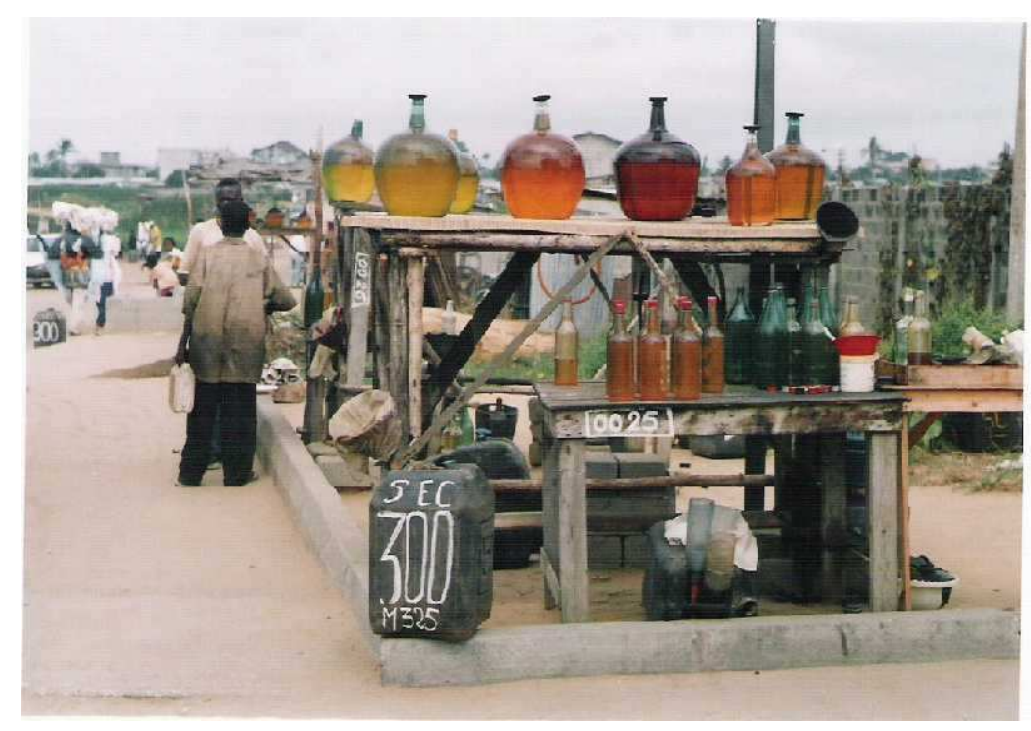

Photo 2: Etalage de vente d'essence frelatée "kpayo" à Cotonou.

La demande accrue en déplacements urbains favorisés, entre autres, par un exode rural accéléré ainsi que l'émergence et la croissance exponentielle de mode de transports privé et individuel ceux-ci se caractérisent par l'usage massif de taxi moto constitués de vieux engins à deux roues communément appelé "Zémidjan". La ville de Cotonou à la date du 30 août 2005, comptait plus de 96.095 " Zémidjan" selon les statistiques obtenues à la Mairie de Cotonou.

\section{Description du site}

Notre étude s'est déroulée à Cotonou, ville la plus importante du Bénin, qui est à la fois capitale économique, centre politique et administratif. Elle s'étend sur une superficie de 7900 hectares environ de part et d'autre d'un chenal et se trouve entre $6^{\circ} 21$ latitude Nord et $6^{\circ} 26$ longitude-Est. Elle est limitée au Nord par le Lac Nokoué, au Sud par l'Océan Atlantique, à l'Ouest par l'arrondissement de Godomey et à l'Est par l'arrondissement d'Agblangandan. Elle est située à une vingtaine de kilomètres environ de la frontière du Nigeria à l'Est et à une centaine environ de celle de Togo à l'Ouest dans la partie méridionale du département de Littoral.
Ce site est coupé en deux par un chenal (ou grau) appelé lagune de Cotonou et qui relie le Lac Nokoué à la mer (Adededji, 1997).

La ville de Cotonou connaît une prédominance des vents du Sud-Ouest de la mousson africaine du Golfe de Guinée (Adededji, 1997).

Les sites étudiés ont été choisis en tenant compte de l'importance du trafic mais aussi dans un souci de quadrillage de toute la ville et de la prise en compte des différents types de carrefours à feu tricolore, à trafic continu avec respect du sens giratoire etc(Houenivo, 2000). La prise des mesures a tenu compte, autant que possible, du sens du vent et des mouvements des véhicules (démarrage et arrêt au feu). Ainsi, avons nous procédé au choix de 4 carrefours réglementés par des feux de signalisation, 3 ronds points, 2 passages à niveau.

\section{Echantillonnage et prétraitement}

Les mesures des gaz $\left(\mathrm{CO}, \mathrm{NO}_{2}\right.$ et $\left.\mathrm{SO}_{2}\right)$ ont été réalisées en $2004-2005$ au niveau de 9 carrefours et 24 boutiques.

\section{Matériel de mesure}

Le détecteur de gaz portatif (Photo 3), permet la détection simultanée de $4 \mathrm{gaz}\left(\mathrm{CO}_{2}\right.$, $\mathrm{CO}, \mathrm{NO}_{2}, \mathrm{SO}_{2}$ ) présents dans l'air. 


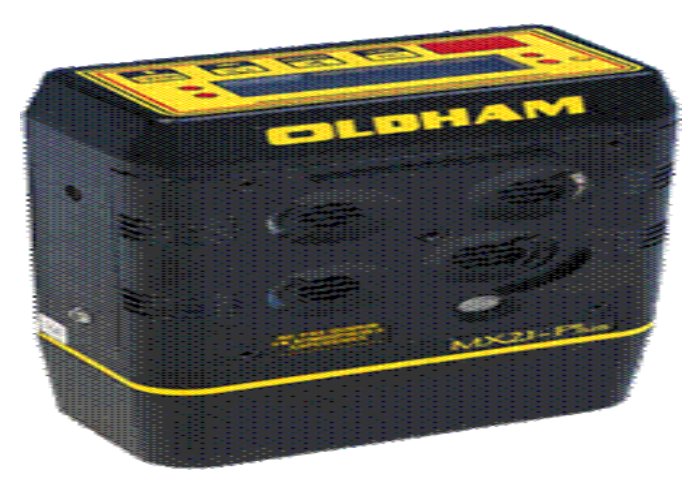

Photo 3: Détecteur de gaz utilisé.

\section{L'échantillonnage du plomb}

L'échantillonnage a été effectué au niveau de deux carrefours: Dantokpa à affluence très élevée et Gbèdjromèdé à flux de circulation et pris au départ comme témoins pour comparaison. Il a été effectué pendant 20 campagnes d'exposition à des heures et jours différents.

\section{Prétraitement}

A la fin des campagnes, nous procédons au lavage des faces exposées avec $500 \mathrm{ml}$ d'eau distillée que nous mettons dans des flacons de 0,5 L. Le dosage du plomb par la méthode de Spectrométrie à absorption atomique est ensuite effectué.

\section{Etude de laboratoire}

Le dosage du plomb s'est fait à l'aide d'un spectromètre à absorption atomique en évaporant la solution de rinçage dans des Béchers de $600 \mathrm{~mL}$ mis à l'étuve à $105^{\circ} \mathrm{C}$ pendant 5 jours.

La minéralisation est faite à l'aide de l'eau régale pendant 2 heures; L'étalonnage est fait à l'aide d'une solution de référence.

\section{RESULTATS}

Observation à l'air libre au niveau des carrefours

\section{Le monoxyde de carbone}

L'observation de la Figure 1 montre que la variation du taux de $\mathrm{CO}$ est fonction de la période de la journée mais aussi des jours ouvrables ou non. Bref, ce taux est fonction de l'intensité du trafic. Par ailleurs, cette même courbe montre que le carrefour Tokpa a les taux les plus élevés avec un maximum de 190 ppm. Les valeurs maximales signalées par la littérature est de 140 ppm et amène de ce fait à considérer ce point comme un point rouge. Après le carrefour Tokpa, arrive le carrefour Midombo avec un maximum de $60 \mathrm{ppm}$.

Le dioxyde de soufre $\left(\mathrm{SO}_{2}\right)$ et le dioxyde d'azote $\left(\mathrm{NO}_{2}\right)$

La Figure 2(a), indique pour le dioxyde de soufre, des taux élevés au carrefour Tokpa avec une valeur maximale de 1,4 ppm de $\mathrm{SO}_{2}$. Ici également le carrefour Midombo vient en $2^{\text {ème }}$ position après Tokpa.

La Figure 2(b), montre l'évolution du dioxyde d'azote $\mathrm{NO}_{2}$ au niveau des différents carrefours. La valeur maximale est obtenue pour le carrefour SOBEBRA (0,4 ppm). Au niveau des carrefours Notre Dame, Tokpa et Midombo les valeurs maximales obtenues sont de 0,3 ppm.

Evolution du taux de Plomb à Gbedjromede et à Tokpa en fonction du temps d'exposition

La quantité du plomb fixée varie avec le temps d'exposition (Figure 5). Elle varie aussi suivant la période d'exposition.

\section{Observation sous abri}

Les résultats des mesures représentés par les courbes ci-dessus indiquent le niveau de pollution de l'air sous abri due aux $\mathrm{CO}$, $\mathrm{NO}_{2}$ et $\mathrm{SO}_{2}$. Les conditions aérologiques ont 
été déterminées à partir des données obtenues à l'ASECNA Cotonou et leur exploitation a permis de cerner les influences possibles qu'elles ont sur le transport et transfert des divers polluants.

\section{- Le CO}

Les mesures de $\mathrm{CO}$ montrent une variation importante de la concentration dans l'atmosphère au niveau des différents carrefours et les différentes enceintes. Ce phénomène s'explique par le fait que l'émission de CO dans l'air est fortement liée à la fluidité de la circulation. Plus le trafic est important, plus les voitures émettent du CO. C'est le cas du carrefour Tokpa dont les différentes mesures effectuées sont nettement supérieures à $30 \mathrm{ppm}$. Ce résultat montre que la concentration de $\mathrm{CO}$ est beaucoup plus importante au niveau des carrefours que dans les boutiques quelle que soit leur localisation. La densité par rapport à l'air du CO est de 0,97 .

La concentration de $\mathrm{CO}$ sous abri au niveau des différents sites n'est pas directement liée à la vitesse du vent mais la concentration du trafic influe beaucoup les taux de $\mathrm{CO}$ dans les boutiques.

\section{- Le $\mathrm{NO}_{2}$ et $\mathrm{Le} \mathrm{SO}_{2}$}

Pour les résultats de $\mathrm{NO}_{2}$, les concentrations obtenues (Fig.5) dépassent 0,3 $\mathrm{mg} / \mathrm{m}^{3}$. Ce taux moyen dépasse la valeur admise par la norme $\left(0,15 \mathrm{mg} / \mathrm{m}^{3}\right)$. La variabilité de ce polluant dans le temps est faible. Malgré la densité de la circulation, la concentration en $\mathrm{NO}_{2}$ reste relativement constante.

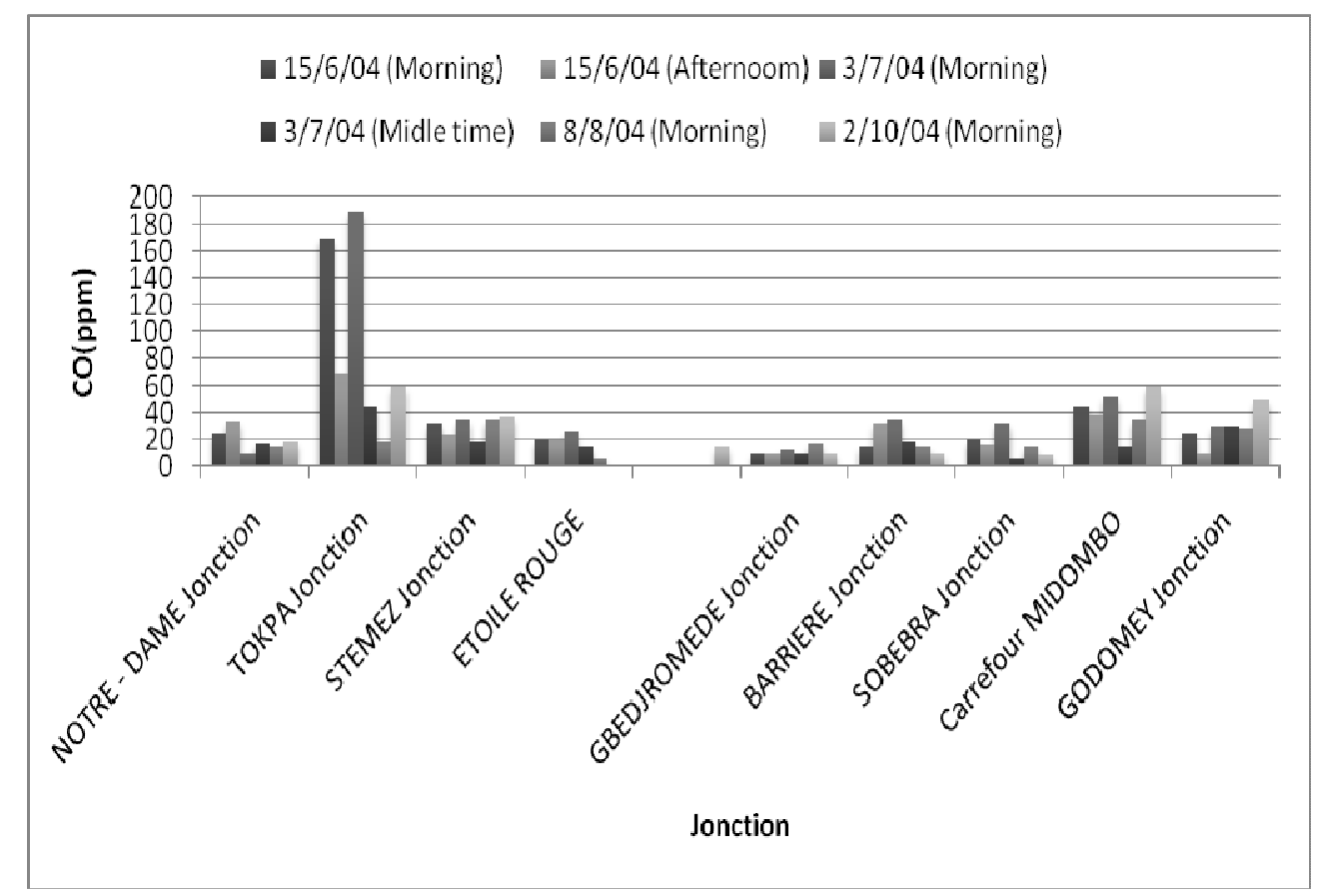

Figure 1: Suivi du monoxyde de carbone des carrefours de Cotonou. 


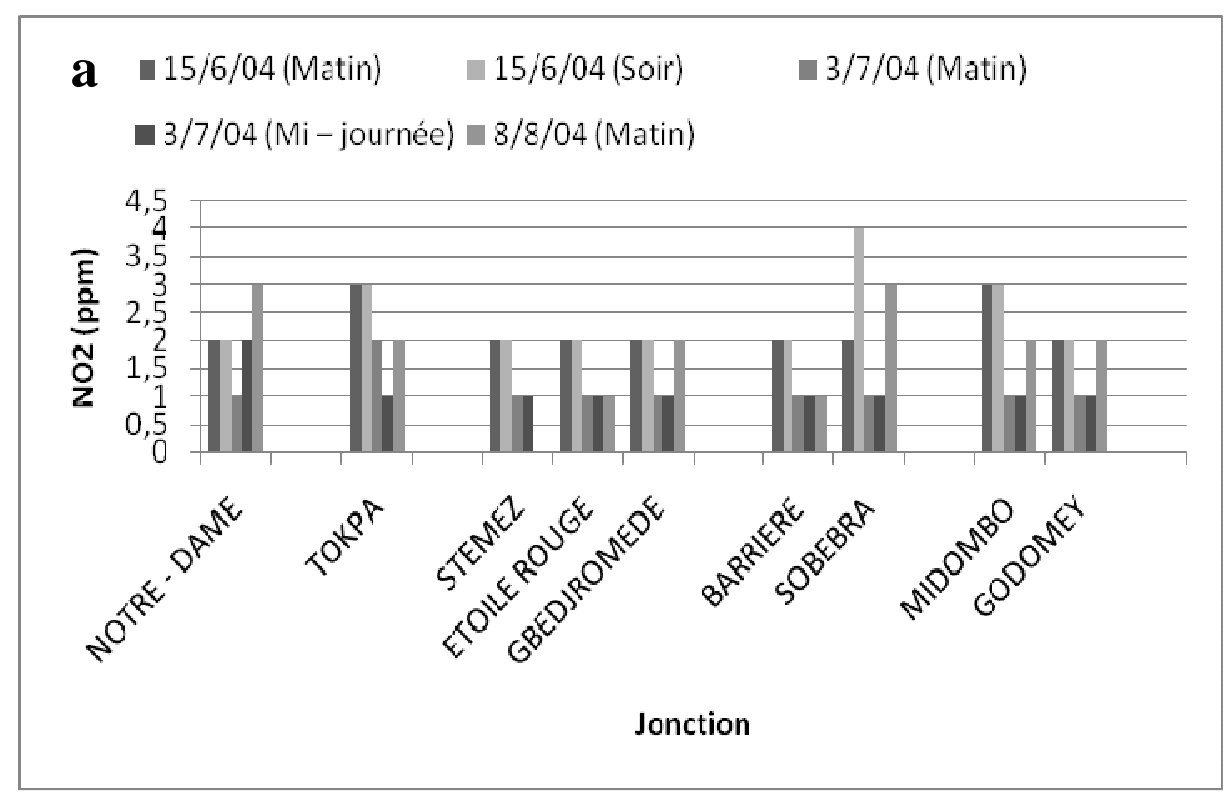

b

\begin{tabular}{|c|c|c|}
\hline ' 5/6;04 (W ornirg) & 口1E/6104 (Afternoom! & 口3/7/04 (M om ng) \\
\hline 口3/7i[4 iMidle :ime) & -8/8104 (Womrg) & ם2/10/04 (N orning) \\
\hline
\end{tabular}

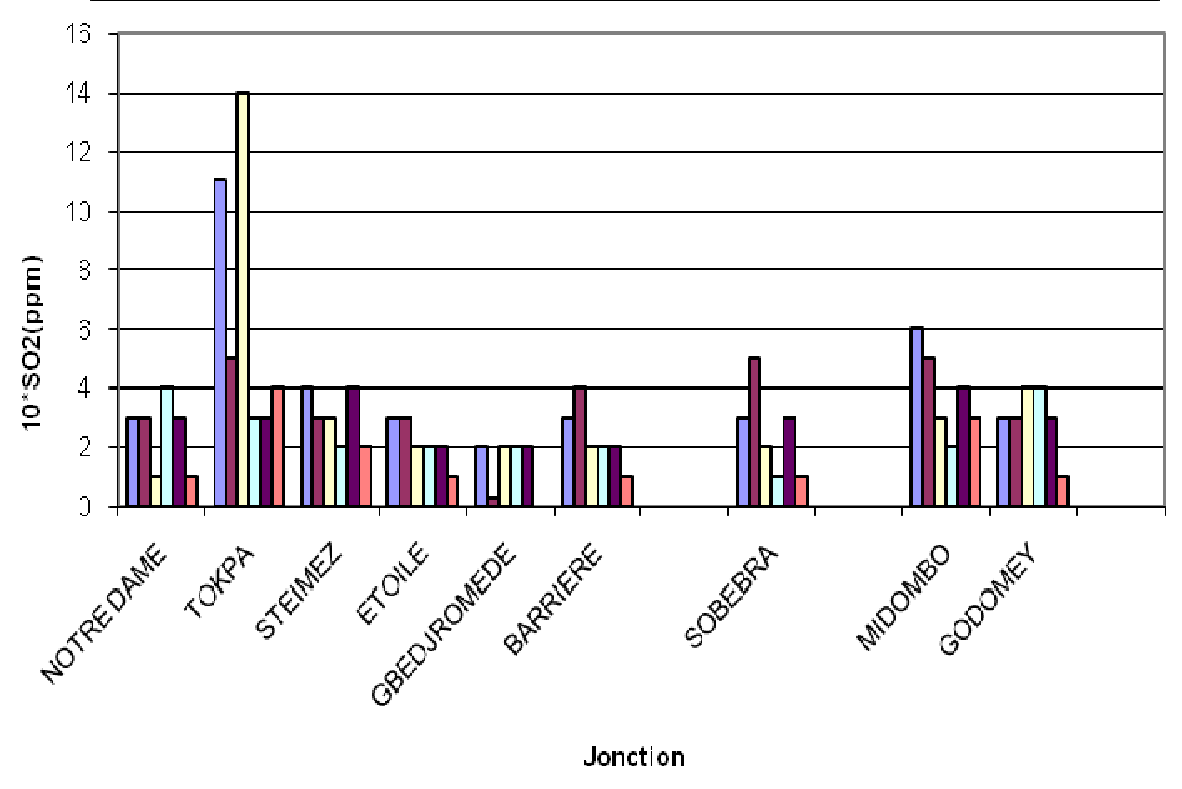

Figure 2: Suivi du dioxyde de soufre (a) et du dioxyde d'azote (b) des carrefours de Cotonou. 


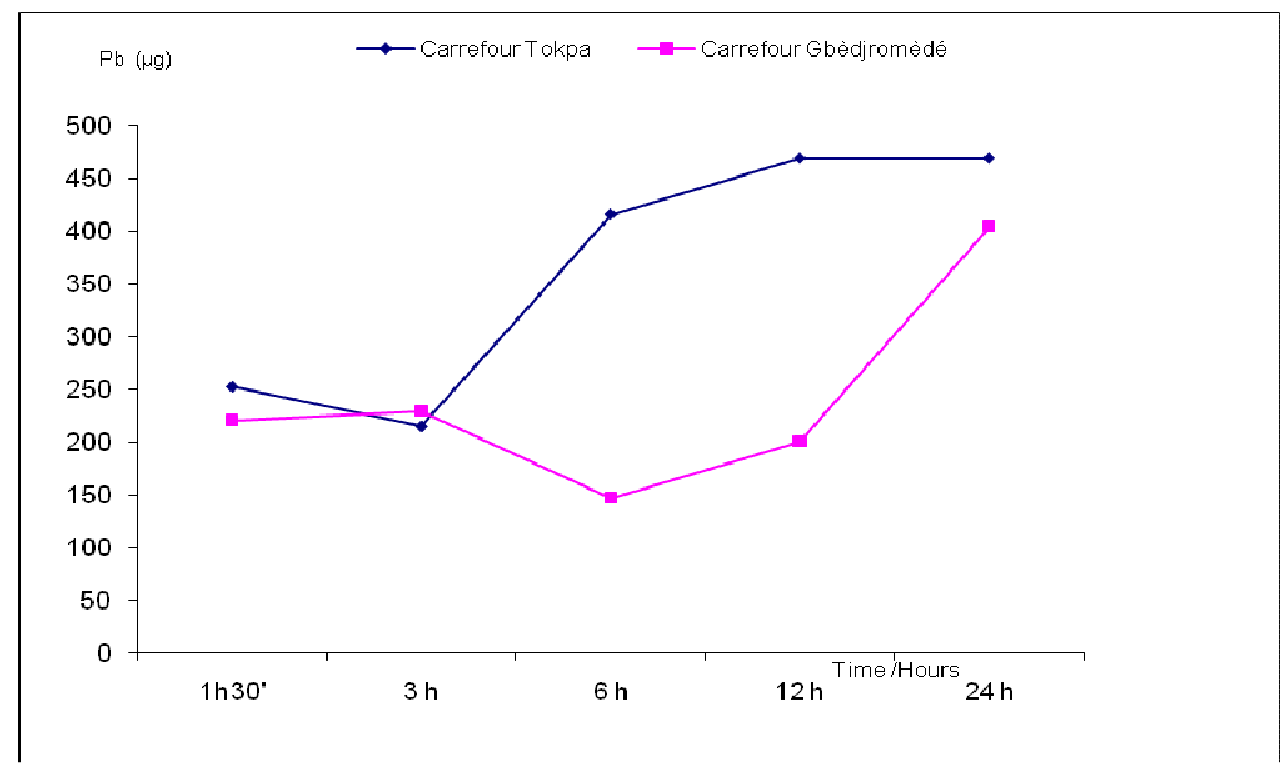

Figure 3: Courbe de suivi du plomb aux carrefours Gbèdjromèdé et Dantokpa selon le temps d'exposition.

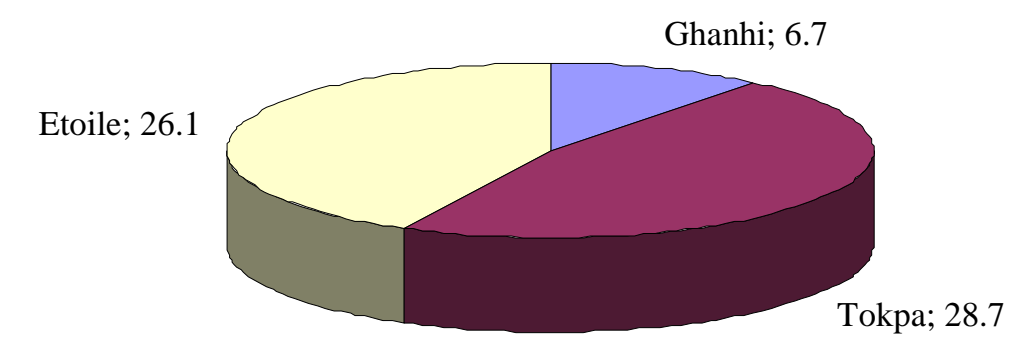

Figure 4: Taux moyen des valeurs maximales de $\mathrm{CO}$ observé sous abris au niveau de quelques carrefours de Cotonou. 


\section{$\mathrm{NO}_{2}\left(\mathrm{mg} / \mathrm{m}^{3}\right)$}

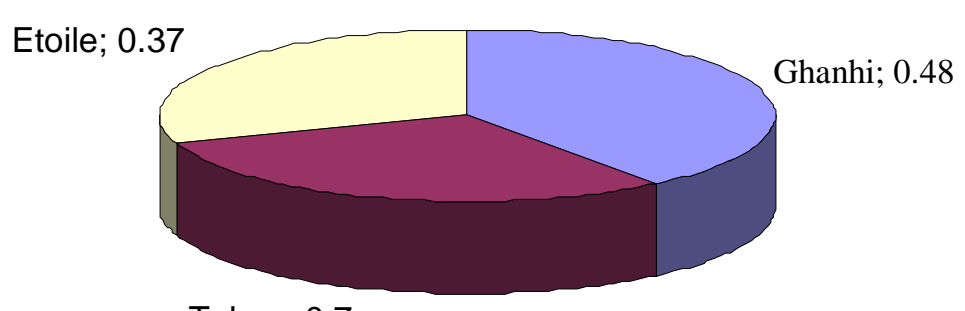

Tokpa; 0.7

Figure 5: Taux moyens des valeurs maximales de $\mathrm{NO}_{2}$.

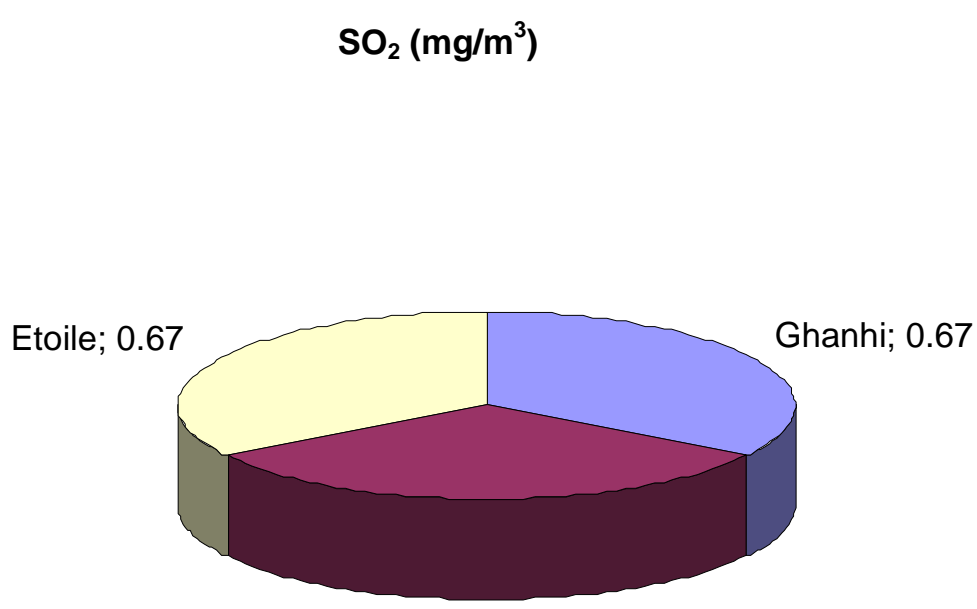

Tokpa; 0.62

Figure 6: Taux moyens des valeurs maximales de $\mathrm{SO}_{2}$ observés sous abris au niveau de quelques carrefours de Cotonou. 


\section{DISCUSSION}

Monoxyde de carbone (CO)

Les valeurs dépassent largement celles prévues par les normes en vigueur au Bénin (10 ppm) ce qui pose en clair le problème de santé vue les effets de ce polluant. Une étude réalisée au cours de l'année 2001 montre un taux de saturation très élevé de $\mathrm{CO}$ dans le sang surtout des conducteurs de taximoto "Zémidjan" (Ahokpè, 2001).

La valeur minimale mesurée est de 6 ppm, obtenue au carrefour Etoile Rouge. Il faut signaler que le maximum observé à ce carrefour est de 46 ppm.

\section{Dioxyde de soufre $\left(\mathrm{SO}_{2}\right)$ et dioxyde d'azote $\left(\mathrm{NO}_{2}\right)$ \\ Les valeurs les plus élevées sont} observées au niveau du carrefour Tokpa (470 $\mu \mathrm{g}$ pour la surface considérée). Néanmoins, Gbèdjromèdé que nous pensons être le témoin à cause de l'affluence faible montre aussi des valeurs relativement élevées $(404,5 \mu \mathrm{g}$ pour la surface considérée). A Tokpa comme à Gbèdjromèdé, les chiffres de résidu de plomb dans l'air sont particulièrement inquiétants. Ces chiffres montrent que la quantité du plomb inhalée augmente en fonction du temps d'exposition des sujets.

Des résultats similaires ont été obtenus à Cotonou en comparaison avec ceux obtenu dans un village de Mono (Avogbé et al., 2005).

\section{Evolution sous abris des polluants $\mathrm{CO}, \mathrm{NO}_{2}$ et $\mathrm{SO}_{2}$}

En considérant les valeurs obtenues sous abris, on note que l'émission de CO dans l'air est fortement liée à la fluidité de la circulation. Plus le trafic est important, plus les voitures émettent du CO. C'est le cas du carrefour Tokpa dont les différentes mesures effectuées sont nettement supérieures à 30 ppm. Ce résultat montre que la concentration de $\mathrm{CO}$ est beaucoup plus importante au niveau des carrefours que dans les boutiques quelle que soit leur localisation. La densité par rapport à l'air du CO est de 0,97.
La concentration de CO sous abri au niveau des différents sites n'est pas directement liée à la vitesse du vent mais la concentration du trafic influe beaucoup les taux de $\mathrm{CO}$ dans les boutiques.

En ce qui concerne le dioxyde de soufre et le dioxyde d'azote, les concentrations sont relativement plus basses dans les boutiques qu'au niveau des carrefours, mais il n'y a pas de différence fondamentale entre les carrefours et les enceintes fermées.

$\mathrm{La}$ concentration en $\mathrm{SO}_{2}$ dans l'atmosphère est faible. Ce faible taux de $\mathrm{SO}_{2}$ s'explique par le fait qu'il n'est pas fortement lié à la circulation (taux de soufre plus faible que celui du carbone dans les hydrocarbures). Le taux de $\mathrm{SO}_{2}$ reste presque constant sous abri. Ces résultats confirment la présence des polluants chimiques liés au transport et le risque sanitaire potentiel qu'encourent les populations aussi bien au niveau des carrefours qu'au niveau des boutiques et autres abris des différents carrefours de la ville de Cotonou.

\section{Le plomb $(\mathbf{P b})$}

La quantité du plomb fixée varie avec le temps d'exposition. Elle varie aussi suivant la période d'exposition. Les valeurs les plus élevées sont observées au niveau du carrefour Tokpa $470 \mu \mathrm{g}$ pour une surface de $1600 \mathrm{~cm}^{2}$ et un temps d'exposition de 12 heures. A Tokpa comme à Gbèdjromèdé, les valeurs de résidus de plomb dans l'air sont particulièrement importantes et dépendant du trafic urbain. Ce résultat est conforme à ceux de Ahokpè (2005).

\section{Conclusion}

Cette étude menée dans la ville de Cotonou a permis d'une part, de mesurer le taux de $\mathrm{CO}$, de $\mathrm{NO}_{2}$, de $\mathrm{SO}_{2}$ et du plomb à l'air libre et sous abri aux différents carrefours et d'autre part, d'étudier l'effet d'exposition au plomb en fonction du temps.

Le travail a permis d'obtenir les résultats suivants : 
$>$ Au niveau des mesures effectuées à l'air libre des carrefours, le taux de monoxyde de carbone (CO) varie de 0,2 à $214,7 \mathrm{mg} / \mathrm{m}^{3}$; le taux de dioxyde d'azote $\left(\mathrm{NO}_{2}\right)$ a donné une valeur minimale de $0,1 \mathrm{mg} / \mathrm{m}^{3}$ et une valeur maximale de $0,52 \mathrm{mg} / \mathrm{m}^{3}$ tandis que les valeurs de dioxyde de soufre $\left(\mathrm{SO}_{2}\right)$ sont comprises entre 0,26 et $3,62 \mathrm{mg} / \mathrm{m}^{3}$.

Pour les mesures effectuées sous abris, on a une valeur minimale de $3,37 \mathrm{mg} / \mathrm{m}^{3}$ et un taux maximum de $29,12 \mathrm{mg} / \mathrm{m}^{3}$ pour le monoxyde de carbone (CO). Le dioxyde d'azote obtenu sous abris varie de 0 à 0,73 $\mathrm{mg} / \mathrm{m}^{3}$. Dans les mêmes conditions, le taux de dioxyde de soufre $\left(\mathrm{SO}_{2}\right)$ s'est situé entre 0 et $0,82 \mathrm{mg} / \mathrm{m}^{3}$.

Les résultats de ce travail montrent au plan spatial que les carrefours Tokpa (à forte affluence) a les taux les plus élevés avec un maximum de 190 ppm $\left(214,7 \mathrm{mg} / \mathrm{m}^{3} \mathrm{CO}\right)$. La valeur maximale signalée par la littérature est de 140 ppm. Les valeurs obtenues pour la plupart des carrefours dépassent largement celles prévues par les normes en vigueur au Bénin $\left(10 \mathrm{mg} / \mathrm{m}^{3}\right.$ CO pour 1 heure d'exposition; MEHU (2001)). La variation du taux de $\mathrm{CO}$ est fonction de l'intensité du trafic.

Les chiffres obtenus, montrent que la quantité du plomb inhalée augmente en fonction du temps d'exposition des différents usagers du trafic et des riverains des sites.

Le travaille a confirmé le rôle principale de pollution de l'air joué par le transport urbain à Cotonou et son impact sanitaire potentiel sur la population de cette ville.

\section{REFERENCES}

Ahokpè AM. 2001. La pollution atmosphérique dans la ville de Cotonou : Évaluation du taux de saturation du sang au Monoxyde de Carbone en fonction du temps d'exposition. Mémoire de fin de formation à la FLASH/UNB, Cotonou, $87 \mathrm{p}$.

Avogbe PH, Ayi-Fanou L, Cachon B, Chabi N, Debende A, Dewaele D, Aissi F, Cazier F, Sanni A. 2005. Ultrafine particulate matter and high-level benzene urban air pollution in relation to oxydative DNA damage. Carcinogenesis, 26(3): 613-620.

Avognon-Houéto L. 1997. Journée de réflexion sur la pollution atmosphérique au Bénin. Rapport de travaux-Cotonou, MEHU, $40 \mathrm{p}$.

Code Permanent. 1994. Environnement et nuisances: pollution de l'air. Code Permanent, pp.447- 479.

David T, Zali O. 1992. Véhicules à moteur et pollution atmosphérique : impact sur la santé publique et mesure d'assainissement. OMS, Genève, VI-VII.

Houenivo T. 2000. L'utilisation des instruments économiques dans la lutte contre la pollution atmosphérique en milieu urbain au Bénin : cas de Cotonou. CAPE, $89 \mathrm{p}$.

MEHU. 2001. Décret n 2001-110 du 04 avril 2001 fixant les normes de qualité de l'air en République du Bénin. Ministère de l'Environnement, de l'Habitat et de l'Urbanisme (MEHU).

Moudachirou M. 2005. Étude de la pollution atmosphérique de la ville de Cotonou. Rapport final, juillet 2005.

PNUE (Programme des Nations Unies pour l'Environnement). 1987. Le Protocole de Montréal relatif à des substances qui appauvrissent la couche d'ozone, Le Secrétariat de l'ozone, PNUE. http://ozone.unep.org/french/Ratification_ status/montreal_protocol.shtml

WHO. 1999. Guideline for Air Quality. World Health Organization: Geneva; 186. 